\title{
Reporte de caso de tuberculosis osteoarticular: dificultad diagnóstica y probable infección primaria
}

\author{
Carlos Diego Valladares Díaz ${ }^{1, a}$, Erika Lucía Pilco Guerra ${ }^{1, a}$, Mary Isabel Ttito Condori ${ }^{1, a}$ \\ RESUMEN
}

Se presenta el caso de un varón de 36 años, procedente de Ancash, Perú, quien acude a un hospital de su localidad por dolor articular en rodilla derecha de tres años de evolución. No se consigue diagnosticar al paciente en dicho hospital y meses después acude al Hospital Nacional Dos de Mayo en la capital, donde posterior al hallazgo de un proceso inflamatorio granulomatoso en una biopsia ósea se inicia una evaluación y tratamiento para tuberculosis osteoarticular, diagnóstico que será posteriormente confirmado por cultivo positivo de líquido sinovial tras un tiempo de enfermedad de más de 45 meses. La presentación atípica, la ausencia de un foco primario y dificultad diagnóstica, en un país donde la tuberculosis es aún endémica, motivaron su descripción para su publicación.

Palabras clave: Tuberculosis osteoarticular; rodilla; artritis infecciosa.

\section{Case report of osteoarticular tuberculosis: diagnostic difficulty and probable primary infection}

\section{ABSTRACT}

We report the case of a 36-year-old male from Ancash, Peru, who attended to a local hospital due to articular pain in the right knee for three years. Diagnosis was not reached in that hospital and months later he visited Hospital Nacional Dos de Mayo in the capital city, where evaluation and treatment for osteoarticular tuberculosis was started after finding a granulomatous inflammatory process in a bone biopsy. Such diagnosis was confirmed later by a positive culture of synovial fluid after a more than 45-month disease period. The atypical presentation, absence of a primary site and diagnostic difficulty of the disease in a country where tuberculosis is still endemic encouraged the publication of this case report.

Keywords: Osteoarticular tuberculosis; knee; infectious arthritis.

1. Estudiante de Medicina Humana.

a. Facultad de Medicina Humana, Universidad de San Martín de Porres. 


\section{INTRODUCCIÓN}

La tuberculosis extrapulmonar es la infección por Mycobacterium tuberculosis a un órgano o sistema diferente al parénquima pulmonar ${ }^{(1)}$. En el Perú, el MINSA

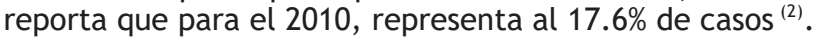

Para su diagnóstico se sugiere tomar biopsia tisular y muestras para baciloscopía y cultivo. En áreas endémicas se logra generalmente con la evaluación clínica y los estudios radiológicos, sin embargo, siempre se recomienda el diagnóstico por biopsia tisular ${ }^{(3)}$.

Presentamos un caso resaltando la dificultad diagnóstica que la tuberculosis osteoarticular supone para el clínico, aún en un país con la prevalencia mencionada.

\section{CASO CLÍNICO}

Paciente varón de 36 años, mestizo, agricultor, procedente de Ancash. Refirió que hace 3 años sufrió una caída con impacto sobre rodilla derecha, a partir de lo cual inició cuadro de dolor moderado tipo punzada, eritema y aumento de volumen de duración de 7 días con recidivas de dos a tres veces por mes. Se automedicó con analgésicos intramusculares por los próximos dos años y medio.
En marzo de 2015 sufrió un nuevo accidente tras el cual no puede extender la rodilla derecha y acude al hospital local de Huaraz, donde fue hospitalizado. Allí se le practicó una punción articular, cuyos resultados se desconocen, y estudios radiográficos que reportan gonartrosis de rodilla derecha y radiografía de tórax normal. Al cuadro se añadió la aparición de una fístula que drenaba líquido purulento. El paciente fue dado de alta con la indicación de acudir al Hospital Nacional Dos de Mayo en Lima.

El 14 de mayo del 2015, el paciente ingresa al servicio de emergencias de HNDM. Al examen físico preferencial presenta aumento de volumen de la rodilla derecha, dolor en la zona de la articulación, fístula con líquido purulento, hipotrofia de cuádriceps derecho, impotencia funcional, y dolor a la flexión y extensión pasiva y activa, con una posición en genuflexión de aproximadamente $130^{\circ}$. Se tomó radiografía de rodilla derecha (Figura 1) que muestra lesiones osteolíticas en epífisis distal de fémur y proximal de tibia con aumento de espacio articular. El hemograma evidenció un proceso inflamatorio agudo activo con leucocitosis a predominio polimorfonuclear. Proteína Creactiva y velocidad de sedimentación aumentados. Se hospitalizó al paciente con el diagnóstico presuntivo de artritis séptica no especificada crónica.

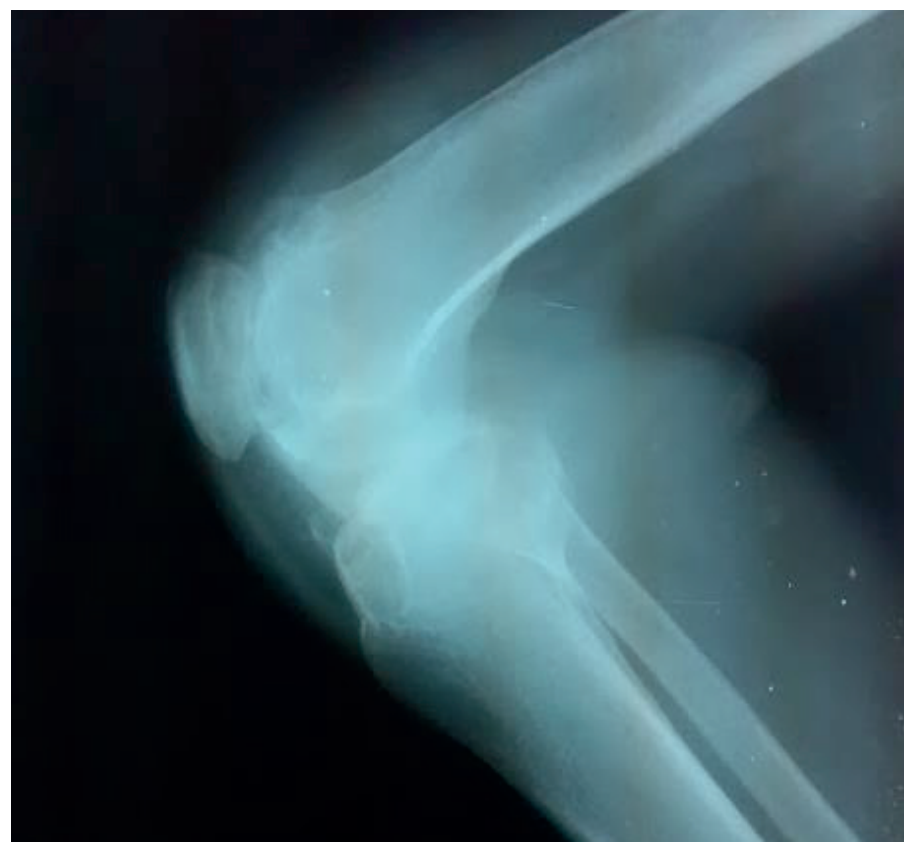

Figura 1. Radiografía de rodilla derecha. Lesiones osteolíticas en epífisis distal de fémur y proximal de tibia con aumento de espacio articular 
Inició terapia antibiótica endovenosa el 18 de mayo con meropenem (1g t.i.d) y vancomicina (1g b.i.d). La radiografía de tórax de control no evidenció neumopatía aguda ni crónica. Se tomó una muestra de secreción de herida, cuyos resultados de cultivo fueron negativos para gérmenes comunes. Se planteó como diagnóstico presuntivo una TBC articular y se buscó un foco pulmonar primario en muestras de esputo para baciloscopía, estas resultaron negativas.

Se realizó una ecografía de partes blandas de rodilla derecha el día 9 de junio donde se observó colección líquida de aspecto abscedado por lo cual el paciente es sometido a una primera intervención quirúrgica el mismo día. Se realizó limpieza quirúrgica, drenaje del absceso y distracción de rodilla derecha. Luego, se cambió el esquema antibiótico endovenoso a ciprofloxacino (200 mg b.i.d) y clindamicina (600 mg t.i.d), y se sostuvo por siete semanas.

El 3 de julio se hizo una nueva limpieza quirúrgica y se tomó una muestra ósea para su análisis anatomo-patológico. Análisis que informó sobre un proceso inflamatorio crónico granulomatoso de tipo tuberculoide, coloración de Ziehl Neelsen (-), Auramina (-) (Figuras 2 y 3). Con base en esto se efectúa otra intervención quirúrgica el 31 de julio donde se realiza artrotomía, limpieza quirúrgica y obtención de muestras de tejido sinovial y secreción articular para cultivo de Mycobacterium. Tras ello se retoma la antibioticoterapia meropenem (1g t.i.d) y vancomicina $(1 \mathrm{~g} \mathrm{b.i.d)}$ por veinte días.

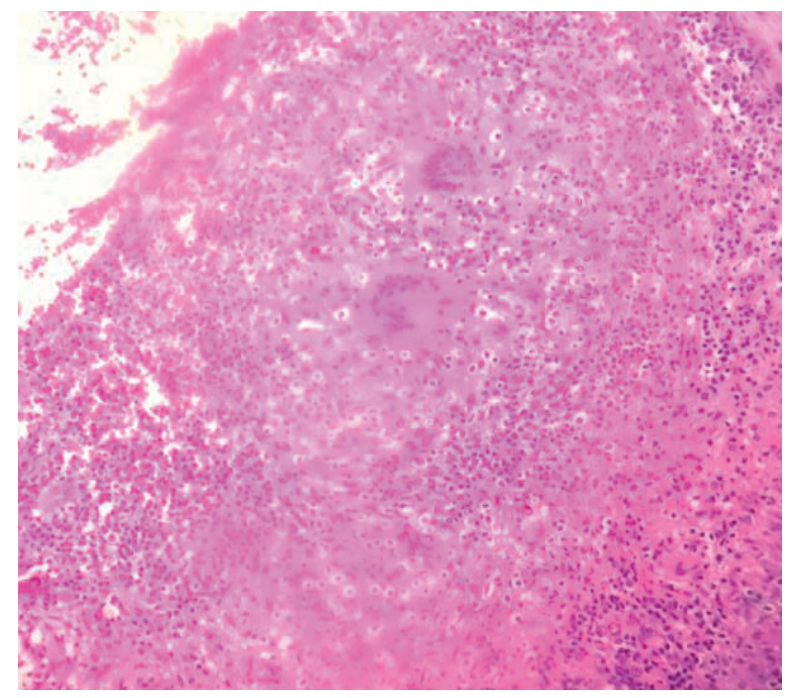

Figura 2. Formaciones granulomatosas en muestra de tejido óseo. Aumento: 40x. Coloración: hematoxilina eosina

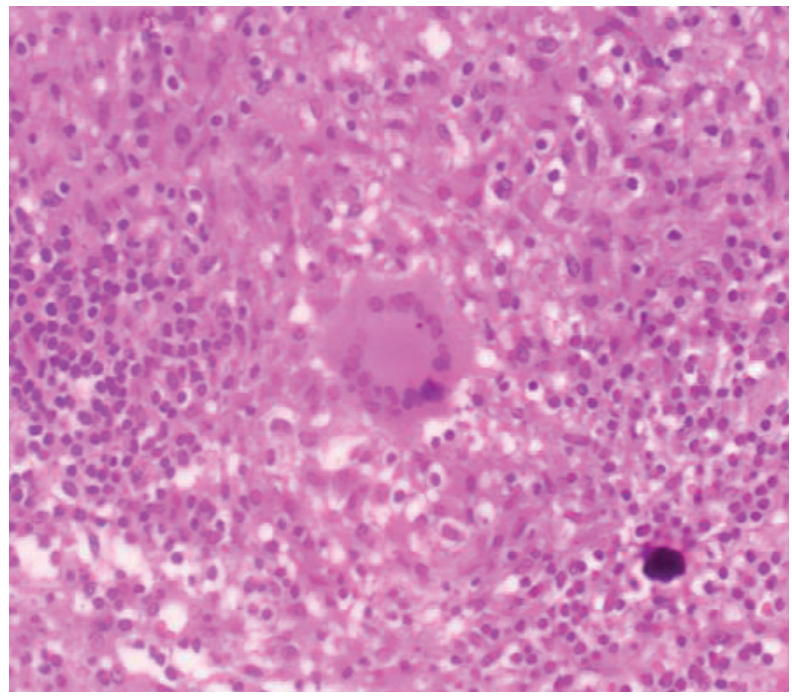

Figura 3. Célula gigante multinucleada en muestra de tejido óseo. Aumento: 100x. Coloración: Hematoxilina eosina 
Basados en la histopatología, se inicia tratamiento para tuberculosis el día 10 de agosto con esquema de isoniazida $(5 \mathrm{mg} / \mathrm{kg})$, rifampicina $(10 \mathrm{mg} / \mathrm{kg})$, pirazinamida $(25 \mathrm{mg} / \mathrm{kg})$ y etambutol $(20 \mathrm{mg} / \mathrm{kg})$.

El resultado del cultivo de secreción articular se obtuvo el 23 de agosto resultando positivo para Mycobacterium tuberculosis. Se concluyó su tratamiento traumatológico con la artrodesis el 27 de agosto. El paciente fue dado de alta el 31 de agosto en su tercera semana de tratamiento antituberculoso, demostrando una evolución favorable.

\section{DISCUSIÓN}

La tuberculosis es la segunda causa de mortalidad infecciosa según la OMS y más del $95 \%$ de los casos y muertes ocurren en zonas en vías de desarrollo. La incidencia anual es de 220,000 casos en nuestro continente y es causa de más de 50,000 muertes $^{(4)}$.

Hacia 2013 la incidencia en Perú fue de 90.3 casos por cada cien mil habitantes. La provincia de Ancash, en torno al caso, tiene 55.3 casos nuevos anuales por 100000 habitantes, calificándose como zona de mediano riesgo para el desarrollo de la infección ${ }^{(5)}$.

La tuberculosis osteoarticular (TBOA) es la tercera locación más común de tuberculosis extrapulmonar, luego de la pleural y ganglionar ${ }^{(6)}$. En caso de la TBOA, se suma al problema de la dificultad diagnóstica, el carácter netamente paucibacilar de esta forma.

La forma de presentación inicial suele ser una osteomielitis en metáfisis, zonas de buena irrigación, que se extiende a espacios articulares ${ }^{(7)}$. Se describe generalmente como una monoartritis con cierta predilección por las articulaciones que soportan peso ${ }^{(8)}$.
Las TBOA suelen presentar una distribución central o de articulaciones periféricas, siendo la tuberculosis espinal lumbar la más frecuente del primer grupo (40\%) ${ }^{(9)}$, y la articulación de la cadera la más frecuente de las periféricas, 12 a $25 \%$ de $\operatorname{casos}^{(6)}$. El diagnóstico de ingreso del paciente es una artritis séptica de rodilla derecha, siendo esta localización el 7 a $8 \%$ de las TBOA ${ }^{(6,9)}$.

Es aún incierto el origen de la infección. Generalmente, las formas extrapulmonares son secundarias a una TB pulmonar diseminada por vía hematógena o linfática; sin embargo, está descrito que hasta $50 \%$ de los casos no presentaría foco pulmonar previo ${ }^{(9)}$.

En el caso presentado, queda en discusión el origen de la artritis tuberculosa, con fuerte sospecha de tratarse de una TBOA primaria. Posiblemente por inoculación directa del germen en piel erosionada ${ }^{(10)}$, considerando el antecedente traumático y radiografías sin signos de TB pulmonar activa o residual. El paciente negó síntomas, diagnóstico o tratamiento previos de tuberculosis pulmonar; alejando la posibilidad de una reactivación de tuberculosis latente.

La presentación es usualmente insidiosa, con dolor, inflamación y disminución del rango articular. Los casos crónicos presentan abscesos o cavidades articulares supurativas, ambos presentes en este caso, en el examen físico y ecografía. Los signos sistémicos estuvieron ausentes, como suele ocurrir ${ }^{(11)}$.

Los cambios radiográficos se describen como inespecíficos, e incluyen edema de partes blandas, osteopenia juxtaarticular, disminución del espacio articular y erosiones subcondrales ${ }^{(11)}$. No suelen ser útiles para el discernimiento etiológico, especialmente en etapa temprana ${ }^{(12)}$. Actualmente la TC y la RM son pilares en el diagnóstico de TBOA ${ }^{(6)}$. Al paciente se le realizó una RM que mostró hallazgos compatibles con artritis séptica (Figura 4).
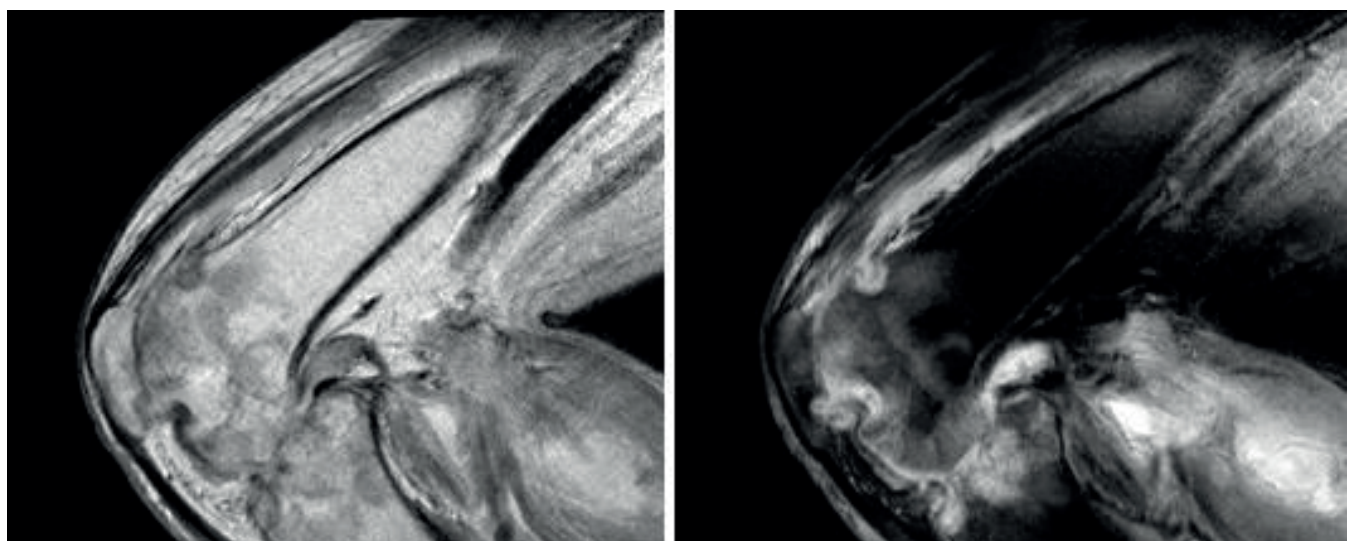

Figura 4. Cavidad articular con hiperseñal heterogénea en T2 y DP, revestida por proliferación sinovial de señal intermedia en DP asociada a erosiones óseas subcondrales en cóndilos femorales, platillos tibial y patelar y edema subcondral subyacente. Hallazgos compatibles con artritis séptica 
La bibliografía recomienda como aproximación diagnóstica basarse en manifestaciones clínicas dependientes del órgano afectado y complementarse con exámenes auxiliares de laboratorio e imágenes. El diagnóstico diferencial incluye enfermedades endémicas según la procedencia ${ }^{(12)}$.

El cultivo de micobacterias es la prueba dorada para el diagnóstico. En TBOA tiene baja tasa de aislamiento por lo que su uso diagnóstico es limitado. Se debe tener en cuenta que el cultivo de líquido sinovial no es definitivo, puede dar resultados positivos hasta en $79 \%$ de los casos ${ }^{(8)}$. En casos de difícil aislamiento, se puede llegar a requerir una biopsia de tejido a partir de la cual cultivar el bacilo, como es nuestro caso.

El estudio histopatológico puede ser problemático ya que los sitios de infección son poco accesibles y hay resistencia de los pacientes a estudios invasivos ${ }^{(6)}$. Este caso es peculiar ya que los granulomas caseificantes respaldan el diagnóstico; así, es recomendable realizar estudios histológicos, aún los estudios microbiológicos sean negativos ${ }^{(7)}$.

Muchos casos en Perú inician tratamiento empírico sin llegar a confirmación histológica o microbiológica. Concretar el diagnóstico de TBOA es un reto que puede tardar incluso años ${ }^{(13)}$

El tratamiento de TBOA requiere de 12 meses. Mientras que el manejo quirúrgico se orienta a mejorar la calidad de vida del paciente debido a lesiones secuelares ${ }^{(9)}$; el tratamiento médico de acuerdo a la norma técnica vigente en nuestro país consiste de una primera fase con isoniacida, Rifampicina, Etambutol y Pirazinamida por 2 meses y una segunda fase de Isoniacida y Rifampicina por 10 meses (12). El desbridamiento y la artrodesis son considerados los tratamientos quirúrgicos principales en fases finales, con buena evolución a una articulación estable y no dolorosa; el desbridamiento completo es crucial y disminuye la recurrencia $^{(14)}$.

Finalmente, como se refleja en nuestro caso, la TBOA puede ser erróneamente diagnosticada por múltiples factores. Se resalta la importancia de no descartar este tipo de manifestación de tuberculosis, especialmente en casos de artritis crónica con estudios comunes negativos, y a pesar de la ausencia de signos de foco primario.

Desafortunadamente las personas de bajos recursos siguen siendo los sujetos más vulnerables a la infección y con mayor riesgo de complicaciones debido a su tardía atención.

\section{REFERENCIAS BIBLIOGRÁFICAS}

1. Espinosa-Gimeno A, Martínez-Sanz J, Asong-Engonga L, Rodríguez-Zapata M. Protocolo diagnóstico y terapéutico de las tuberculosis extrapulmonares. Medicine - Programa de Formación Médica Continuada Acreditado [Internet]. 2014 [cited 10 October 2015];11(52):3091-3097. Available from: http://www.sciencedirect.com/science/article/pii/ S0304541214707450

2. Ministerio de Salud. Estrategia Sanitaria Nacional de Prevencion y Control de la Tuberculosis. Lima: Ministerio de Salud; 2010. $1-2$.
3. Tuli S. General Principles of Osteoarticular Tuberculosis. Clinical Orthopaedics \& Related Research [Internet]. 2002 [cited 12 October 2015]; 398:11-19. Available from:http:// journals. Iww.com/corr/Abstract/2002/05000/General_ Principles_of_Osteoarticular_Tuberculosis.3.aspx

4. Ministerio de Salud. Situación de la Tuberculosis en el Perú. Lima: Direccion General de Salud de las Personas; 2008. 2-78.

5. Alarcón A. Situación de la Tuberculosis en el Perú y Política Nacional para su Control. Arequipa: Ministerio de Salud; 2014. 1-68.

6. Chen S, Zhao L, Dong W, Gu Y, Li Y, Dong L, et al. The Clinical Features and Bacteriological Characterizations of Bone and Joint Tuberculosis in China. Sci Rep. [Internet]. 2015 [cited 10 October 2015];5:11084. Available from: http://www.ncbi. nlm.nih.gov/pubmed/26053666

7. Iseman MD. A clinician's guide to tuberculosis. Philadelphia: Lippincott, Williams \& Wilkins; 2000.162

8. Kramer N, Rosenstein E. Rheumatologic manifestations of tuberculosis. Bull Rheum Dis. 1997; 46(3):5-8.

9. Lidder $\mathrm{S}$, Lang $\mathrm{K}$, Haroon $\mathrm{M}$, Shahidi $\mathrm{M}$, El-Guindi $\mathrm{M}$. Tuberculosis of the knee .Orthopedic Reviews. 2009; 1(2):e24.

10. Pasion E, Leung J. TB arthritis. Current Orthopaedics [Internet]. 2000 [cited 5 October 2015]; 14(3):197-204. Available from: http://www.sciencedirect.com/science/ article/pii/S0268089000901063

11. Watts HG, Lifeso RM. Tuberculosis of bones and joints. J Bone Joint Surg Am. 1996; 78:288-98.

12. Ministerio de Salud. Norma Técnica de Salud para la Atención Integral de las Personas Afectadas por Tuberculosis. Lima: Ministerio de Salud; 2013. 21-30.

13. Yao DC, Sartoris DJ. Musculoskeletal tuberculosis. Radiol Clin North Am. 1995; 33(4):679-89.

14. Tang X, Zhu J, Li Q, Chen G, Fu W, Li J. Knee arthrodesis using a unilateral external fixator combined with crossed cannulated screws for the treatment of end-stage tuberculosis of the knee. BMC Musculoskelet Disord. [Internet]. 2015 [cited 7 October 2015];16:197. Available from: http://www.ncbi.nlm. nih.gov/pubmed/26286256

Fuentes de financiamiento:

Este artículo ha sido financiado por los autores.

Conflictos de interés:

Los autores declaran no tener ningún conflicto de interés.

Correspondencia:

Erika Lucía Pilco Guerra

Dirección: Av. Aviación 2382, Dpto. 501, San Borja.

Teléfono: 942958972

Correo electrónico: erika.pilco2@gmail.com

Recibido: 16 de junio de 2016 Aprobado: 08 de julio de 2016 